\title{
Traffic Signal Optimization under Connected-Vehicle Environment: An Overview
}

\author{
Jindong Wang, ${ }^{1,2}$ Shengchuan Jiang $\mathbb{D},{ }^{1}$ Yue Qiu $\mathbb{D},{ }^{1}$ Yang Zhang, ${ }^{3}$ Jianguo Ying, ${ }^{2}$ \\ and Yuchuan Du $\mathbb{D}^{1}$ \\ ${ }^{1}$ Key Laboratory of Road and Traffic Engineering of the Ministry of Education, Tongji University, Shanghai, China \\ ${ }^{2}$ Jinqiao (Group) Co., Ltd., Shanghai, China \\ ${ }^{3}$ Shanghai Urban-Rural Construction and Transportation Development Institute, Shanghai, China
}

Correspondence should be addressed to Shengchuan Jiang; shengchuanjiang@tongji.edu.cn

Received 10 May 2021; Revised 19 July 2021; Accepted 3 August 2021; Published 11 August 2021

Academic Editor: Chi-Hua Chen

Copyright (c) 2021 Jindong Wang et al. This is an open access article distributed under the Creative Commons Attribution License, which permits unrestricted use, distribution, and reproduction in any medium, provided the original work is properly cited.

Traffic signal optimization is a significant means for smoothing urban traffic flow. However, the operation of traffic signals is currently seriously constrained by the data available from traditional point detectors. In recent years, an emerging technology, connected vehicle $(\mathrm{CV})$, which can percept the overall traffic environment in real time, has drawn researchers' attention. With the new data source, traffic controllers should be able to make smarter decisions. A lot of work has been done to develop a new traffic signal control pattern under connected-vehicle environment. This paper provides a comprehensive review of these studies, aiming at sketching out the state of the arts in this research field. Several basic control problems, communication, control input, and objectives, are briefly introduced. The commonly used optimization models for this problem are summarized into three types: rule-based models, mathematical programming-based models, and artificial intelligence-based models. Then some major technical issues are discussed in detail. Finally, we raise the limitation of the existing studies and give our perspectives of the future research directions.

\section{Introduction}

The recent years have witnessed an exponential increase in vehicular traffic in urban areas. As a result, a variety of challenges have emerged, including traffic congestion, energy consumption, traffic safety risk, and pollutant emissions. As reported by the Texas A\&M Transportation Institute and INRIX, Americans spent 6.9 billion hours of extra time and purchased 3.1 billion gallons of extra fuel due to congestion in 2014, causing average cost of $\$ 960$ for every autocommuter compared to an inflation-adjusted $\$ 400$ in 1982 [1]. In another report, the total number of crashes in 2015 was over 6.2 million, 3.8\% higher than that in the previous year [2]. For the same period, the ratio of total carbon dioxide $\left(\mathrm{CO}_{2}\right)$ emissions attributable to transportation reached $32.1 \%$, about $3 \%$ higher than the ratio in the year of 1990 [3]. With the rapid motorization, especially in developing countries, it is expected that $70 \%$ of the people worldwide will live in cities [4] and the number of operating vehicles around the world will at least double by 2050 [5]. Unless effective measures are taken, these problems, therefore, may deteriorate in the future.

To solve the aforementioned problems, one may consider expanding the road facilities to satisfy the increasing travel demand. But a more feasible and low-cost solution is to optimize the traffic signal to balance the traffic load and decrease waiting times, based on the fact that road intersection is the bottleneck of the traffic network and thus one of the major contributors to traffic inefficiency. Since the first prototype colored traffic signal light was installed in England in 1868 [6], signal control systems have experienced ongoing development. Nowadays, the in-use traffic control strategies can be categorized into three types: (1) fixed-time, (2) actuated, and (3) adaptive control. 
(1) Fixed-time control: Fixed-time control predefines a static and repeating sequence of phases and durations based on historical data to serve different times of the day (TOD) to address the demand fluctuation. For the reason that the timing plan is designed based on historical data in an offline manner, this control strategy is also known as offline control. The underlying assumption of fixed-time control is that the traffic demand remains unchanged within the entire time period of a timing plan. Examples include Webster's method [6] and its extensions, SIGSET [7], SIGCAP [8] for isolated control together with MAXBAND [9] and its extensions, TRANSYT [10], and MULTIBAND [11] for coordinated control.

(2) Actuated control: Actuated control detects the dynamic traffic demand to modify a fixed timing plan by occasionally skipping a phase if no vehicle is present or shortening a phase when vehicles are not being served. Actuated control and the following adaptive control enable traffic signal controllers to make use of real-time traffic information (e.g., vehicle counts, lane volume, and lane occupancy), so the two control methods belong to online control. MOVA [12] is a typical actuated control system which uses the traffic data upstream of the stop-line to implement control logic. In the USA, actuated signal control is widely adopted according to the signal control guidelines recommended by the Federal Highway Administration [13].

(3) Adaptive control: Adaptive timing plans attempt to continuously change their signal phases and timings in response to real-time traffic conditions. Therefore, this method is more flexible than actuated control. Adaptive control was first proposed as early as 1960s [14]. The early studies and implementations include SCOOT [15], SCATS [16], PRODYN [17], OPAC [18], and UTOPIA [19]. The recently developed systems such as RHODES [20] can realize proactive control by predicting traffic demands at a downstream intersection and optimizing lost times on a global scale.

The advantage of fixed-time control systems lies in calculation simplicity as well as lower hardware requirements. But the pretimed timing plans cannot capture occasional events such as traffic accidents and road closure. In addition, the variation trend of traffic volume is not exactly identical on different days. Therefore, actuated and adaptive control systems are increasingly adopted in metropolises in order to deal with the downsides of fixed-time control. However, there are still several unsolved problems. All the aforementioned actuated and adaptive signal control systems collect real-time traffic data from infrastructurebased sensors, for example, loop detectors, ultrasonic detectors, or video detectors, which can only conduct point detection and estimate traffic states based on very limited information, such as vehicle counts or temporal gap between consecutive vehicles. Inductive loop is broken down frequently in practical application, while the ultrasonic sensor and video detector are very sensitive to the weather, resulting in degraded system performance. Moreover, installation and maintenance of these detectors require frequent and costly investments. In summary, the existing systems are not able to collect traffic information comprehensively, steadily, and at low cost.

In this context, Intelligent Transportation Systems (ITS) are considered as the key for enhancing the capacity of the traffic control systems. With the advances in wireless communication technology, vehicular ad hoc networks (VANETs) are created by applying the principles of mobile ad hoc networks (MANETs) to the domain of vehicles and become a significant part of ITS framework [21]. In VANETs, vehicles are able to communicate with each other (vehicle-to-vehicle communication, V2V) and with the infrastructure (vehicle-to-infrastructure communication, V2I) through dedicated short-range communications (DSRC) (or any other wireless networking technology) and are referred to as connected vehicles (CVs). Data from CVs provide a complete picture of the vehicle states including location, speed, acceleration, and other vehicle data. Compared with the conventional point detection sensors, the traffic information provided by CVs is also steadier and more persistent because fault of an individual vehicle will only slightly decrease the penetration rate and the system can still provide relatively accurate information. In addition, such a system saves installation and maintenance cost of specific traffic flow detectors. Based on the new source of data, traffic controllers should be able to make smarter decisions. In the last decade, researchers began to explore the benefits of using such information. For example, Gradinescu et al. [22] designed an adaptive traffic light system relying on wireless communication between vehicles and traffic light controller, where the algorithm is still based on the traditional Webster's signal timing formula but all the information needed is collected by VANETs; Kari et al. [23] proposed an online adaptive traffic signal control (ATSC) strategy based on CV technology, which is capable of adjusting traffic light settings, including green splits and phase sequence in response to the variations in traffic demand and arrival pattern. The studies show the potential of CV technology used in traffic signal optimization.

Another trend, as is well known, is the development of autonomous driving technology. The connected vehicles combined with autonomous driving technology, so-called connected and autonomous vehicles (CAVs), is being hailed by both academia and industry as the revolution of human mobility. The National Highway Traffic Safety Administration (NHTSA) defined vehicle automation into five levels, ranging from vehicles that do not have any automated control functions (level 0 ) through fully automated vehicles (level 4) [24]. In a long time of the future, vehicles with different technical levels may coexist in the transportation systems. So how to improve traffic operations in a road environment containing both traditional vehicles and autonomous vehicles is a key problem referring to traffic 
control in the near future. On the other hand, vehicle type can be easily identified through the information provided by connected vehicles. Hence, traffic signal priority (TSP) can be implemented in new ways and, therefore, is equally worth of focusing on nowadays.

The major contribution of the presented paper is to give a comprehensive review of the state-of-the-art techniques with respect to traffic signal optimization under connectedvehicle environment. It should be noted that traffic control generally includes controlling the traffic at intersections, ramp-metering, and variable speed limits as well as route guidance, but the scope of the review is limited in intersection signal control. Information about ramp-metering under CV environment is available in other literatures such as $[25,26]$. In the remainder of the paper, we first introduce some fundamental problems referring to V2X-based traffic signal control including communication systems, data requests, and control objectives. Then optimization models are reviewed according to the model paradigms. The following is the discussion about some major technical issues. Although immense amounts of concrete research focused on this topic, there are still many common problems which remain to be solved. In the last section, we will raise several remaining problems and state our viewpoints about the future research directions.

\section{V2X-Based Traffic Signal Control System}

2.1. Basics of Vehicular Communication. CV communication is an emerging technological framework that aims at direct data transmission between vehicles to vehicles (V2V) and vehicles to road infrastructure (V2I) using wireless technology. It is generally known that CV communication standards consist of three components: (1) IEEE 1609 "Family of Trial-Use Standards for Wireless Access in Vehicular Environments (WAVE)" [27], (2) IEEE 802.11p "Standard for Information Technology" [28], and (3) Society of Automotive Engineers International (SAE) J2735 "DSRC Message Set Dictionary” [29]. These standards are sometimes called WAVE/DSRC for short. The IEEE 1609 family defines an overall structure of the WAVE interface. IEEE 802.11p deals with the multichannel operations of the MAC layer. SAE J2735 defines the framework of DSRC messages, for example, here-I-am (HIA), a-la-carte (ALC), and signal phase and timing (SPaT) messages, to ensure interoperability among any possible CV applications. For safety applications, SAE J2735 also defines the Basic Safety Message (BSM), which is the most fundamental building block that enables proximity awareness. Such framework includes the elements and the usages of possible messages categorized based on the types of applications.

The unique difference of WAVE/DSRC as compared to existing wireless communication standards is that it does not require an authentication procedure. That is, under existing wireless LAN protocols, like IEEE $802.11 \mathrm{a} / \mathrm{b} / \mathrm{g}$, a mobile node (i.e., a laptop or a smart phone) must be identified by an access point (AP) to join the network. However, these identification steps normally take a few seconds or even minutes and are thus not suitable for a mobile network composed of fast-moving vehicles. Thus, by omitting such identification steps, WAVE/DSRC enables quick connections between transceivers.

The channel operation of WAVE/DSRC is also of interest. The DSRC has a $75 \mathrm{MHz}$ frequency spectrum at $5.9 \mathrm{GHz}$ frequency band. The spectrum is divided every $10 \mathrm{MHz}$, resulting in seven different channels from 172 to 184 [28]. Channel 178 is called a control channel (CCH), and the other channels are called service channels (SCHs), except for the two channels at both ends that are reserved for future use. While the $\mathrm{CCH}$ is dedicated to the transmission of control messages such as beaconing or urgent safety-related messages, the SCHs are designed for exchanging any data packets including vehicular mobility information or commercial services. Therefore, the control messages or safetyrelated data that are transmitted through the $\mathrm{CCH}$ are not affected by data transmission through a $\mathrm{SCH}$, thereby enabling the system to be suitable for the fast-moving mobile network.

On the basis of the above technologies, each vehicle transmits its temporary ID, location, speed, heading, lateral and longitudinal acceleration, brake system status, and vehicle size to surrounding vehicles and the infrastructure. By "listening" to these messages, a signal controller could gain a more comprehensive understanding of the movements of nearby vehicles than with traditional point detectors (e.g., loop detectors). Substantial improvement was seen in several real-world pilot projects such as Audi [30] and BMW [31].

\subsection{Data Requirement of Traffic Signal Control. Traffic signal} optimization algorithms need appropriate input including both static information about road facilities and real-time dynamic traffic states. Static information is usually preset in optimization models, while dynamic information depends on real-time communication between vehicles to vehicles and vehicles to infrastructures. In the reviewed literatures, the used real-time traffic information can be categorized into aggregative and individual vehicle information. The advantage of aggregative information lies in modest resource requirements such as average transmission content and computation power. In contrast, the upside of individual information is fine-grained considerations of the optimization problem. Examples of aggregative information include total vehicle counts, equipped vehicle ratio [32], and queue length in the lanes related to each traffic lights phase $[33,34]$. Required individual vehicle information includes real-time location [35], speed [36], heading [37], and acceleration [38] as well as vehicle type [39] of each vehicle within a certain spatial range. Sometimes, weather and minor events [40] are also taken into account to improve the operations.

2.3. Control Objectives. In the traffic signal timing optimization process, one or more of the measures of effectiveness (MOEs) are optimized under certain constraints to determine appropriate signal timing parameters including phase plans, cycle lengths, green splits, and offsets. Different 
objective functions and their combinations are used to define the problem. Therefore, proper selection of the objective function for signal timing optimization is a very important task. Minimizing time lost in the intersections, such as average delay, average waiting time, or travel time, is the most commonly selected objective function [41-43]. Some of the studies also considered the variance of waiting time to improve fairness [44]. Several studies conducted queue-based optimization, that is, minimizing average or maximum queue length $[34,45]$. In order to consider the environmental influence, decreasing pollutant emissions can be imported as an objective function [46]. It is noted that the mentioned objectives are not independent but interrelated with each other. For example, it is discovered that minimizing total delay can also minimize the total number of stops and, hence, is more suitable for implementation [47]. To consider multiple factors at the same time, special performance index (PI), which is usually defined as the weighted sum of several variables, can also be used in optimization model. For instance, Goodall et al. optimized signal timing plans by minimizing a combination of delay, stops, and decelerations [37]. There are also studies with the objective function considering the minimization of fuel consumption such as [48].

In the reviewed literatures, an unsolved problem is the selection of objective function under different application scenarios. The problem has been stated in a recent research [49].

\section{Optimization Models for Traffic Signal Control under CV Environment}

The optimization model, as the core part of a traffic signal control system, outputs the timing plan including cycle lengths and phase distribution and thus needs to be well designed to improve the control effect. In this section, we summarize the proposed optimization models into three types: rule-based models, mathematical programming-based models, and artificial intelligence-based models. Uncategorized models can be found in other models.

3.1. Rule-Based Models. Rule-based models are defined as the models which determine the control parameters through specific equations built based on certain optimization criteria. This type of models mostly inherited traditional fixtime control algorithms, replacing the historical traffic data with real-time traffic states awareness under the $\mathrm{CV}$ environment.

For isolated intersections, the classical approach to determine cycle lengths is based on the well-known Webster's equation [50], which is a function of lost times and critical flow ratios, for minimum delay cycle lengths:

$$
C=\frac{1.5 \cdot L+5}{1-\left(1 / X_{C}\right) \cdot \sum_{i=1}^{n}\left(v_{i} / s_{i}\right)},
$$

where $C$ is the optimum cycle length; $L$ is the sum of lost times for all phases in a cycle; $n$ is the number of critical lane groups; $1 / X_{C}$ is the desired degree of intersection utilization (1.0 for operation at full capacity). Then the green time is then distributed according to the lost time of all phases:

$$
G=C-\sum_{i=1}^{n}\left(Y_{i}-L_{i}\right),
$$

where $G$ is the green time; $Y_{i}$ is the yellow time per phase $i ; L_{i}$ is the lost time per phase $i$.

Webster's method is modified to adapt the CV environment in several studies. A typical example is the CATS system proposed by Maslekar et al. [51]. In this system, the cycle time is formulated by a modified Webster's equation:

$$
C=\frac{1.5 \cdot L+5}{1-\sum(D / \mathrm{Ln})}
$$

where $D / \operatorname{Ln}$ is the ratio of density $D$ in the cluster to the length Ln of the cluster. A clustering algorithm is defined, which will assist in estimating density $D$ of vehicles approaching an intersection. Moreover, two approaches, that is, C-DRIVE and MC-DRIVE, were adopted and compared for density estimation at intersections. The proposed system is compared with a classic pretimed control system and an adaptive control system by simulation. The simulations also show that the data convergence time and the communication delay between vehicles and traffic signals do not decrease the efficiency of the system. Similar studies include [22, 41, 52].

More extensions reflect in estimating the traffic states in the near future and developing network-wide control. Lee et al. [32] proposed a cumulative travel-time responsive (CTR) real-time intersection control algorithm. The core of the algorithm is based on a stochastic state estimation technique utilizing Kalman filtering that is used in estimating the cumulative travel times under imperfect market penetration rates at every update interval. Compared to an optimized actuated control algorithm, the proposed CTR algorithm improved the total delay time and average speed of the intersection by 34 and 36\%, respectively, at $100 \%$ market penetration. Bani Younes et al. [53] designed an ITL scheduling algorithm (ITLC) which utilizes vehicular ad hoc communications technology to gather the real-time traffic characteristics of all competing flows of traffic at each signalized road intersection. Further, the ATL algorithm was introduced for open-network control scenarios aiming at high traffic fluency for the arterial flows. Evaluations revealed that the ATL algorithm decreases the average queuing delay at each traffic light by $10 \%$ compared with previously introduced traffic scheduling systems. Lin et al. [54] proposed an algorithm that limits the boundary flow of a road network based on MFD and controls the maximum queuing length of each boundary section to avoid the overflow phenomenon.

Rule-based models have the advantages of conciseness and computational convenience. However, the coarsegrained modeling usually contains too much simplification, which compromises the accuracy of the formulation.

\subsection{Mathematical Programming-Based Models.}

Mathematical programming, which means the selection of a best element with regard to some criterion from the feasible region [55], is the most commonly used method to optimize the timing plan of intersection signal control. As 
a classic paradigm in the field of transportation research, considerable studies have made great contributions in this regard. Some representative studies are reviewed below.

Zhu and Ukkusuri [56] developed a linear programming formulation for autonomous intersection control (LPAIC) accounting for traffic dynamics within a $\mathrm{CV}$ environment. Firstly, a lane-based bilevel optimization model is introduced to propagate traffic flows in the network, accounting for dynamic departure time, dynamic route choice, and autonomous intersection control in the context of system optimum network model. Then the bilevel optimization model is transformed to the linear programming formulation by relaxing the nonlinear constraints with a set of linear inequalities. Also, the LPAIC formulation propagates traffic flow consistently as LTM and CTM. The control system is tested in both isolated and grid network scenarios. The simulation result shows that the proposed LPAIC algorithm produces lower total travel time in different $\mathrm{V} / \mathrm{C}$ than an actuated longest queue first signal control algorithm.

Feng et al. [57] presented a real-time adaptive signal phase allocation algorithm using connected-vehicle data. The proposed algorithm optimizes the phase sequence and duration by solving a two-level optimization problem to minimize total vehicle delay and queue length. A real-world intersection is modeled in VISSIM to validate the algorithms. The results show that the proposed control algorithm outperforms actuated control by reducing total delay by as much as $16.33 \%$ in a high penetration rate case.

Li et al. [48] develop a modeling framework for optimizing the timing of a set of traffic signals by considering individual vehicle characteristics such as fuel consumption and travel time. The proposed strategy applies the intelligent driving model (IDM) to predict vehicle trajectories under the connected-vehicle environment. The resulting model is a mixed-integer nonlinear program. Mixed-integer linear or nonlinear model is also frequently adopted in numerous studies, especially studies referring to priority control [39, 58-60].

Priemer and Friedrich [61] presented a traffic control strategy which is phase-based as traditional traffic signal control methods but operates without common parameters like cycle times, offsets, or other fixed timings. In each discrete interval of $5 \mathrm{~s}$, the control algorithm forecasts the future queue length for the next 20 seconds by listening to the received vehicles position and speed data. Within each of these optimization horizons, the method determines the optimal phase sequence in order to reduce the total queue length at an intersection by using the methods of dynamic programming (DP) and complete enumeration (CE) algorithm. Approximate dynamic programming, a variant DP method which allows the controller to learn from its own performance progressively, is adopted in a study by Cai et al. [62]. This study presents a method, VICAC, which combines travel-time estimation and adaptive traffic signal control. Li and Ban [63] decomposed the signal optimization and coordination problem into two levels: an intersection level to optimize phase durations using dynamic programming (DP) and a corridor level to optimize the offsets of all intersections.
Pandit et al. [36] formulate the vehicular traffic signal control problem as a job scheduling problem on processors, with jobs corresponding to platoons of vehicles. Then the oldest job first (OJF) algorithm is used to minimize the delay across the intersection. The evaluation result shows that the algorithm reduces the delays experienced by vehicles as they pass through the intersection under light and medium traffic loads as compared with vehicle-actuated methods, Webster's method, and pretimed signal control methods.

For mathematical programming, the greatest challenge is the nonlinearities of objective functions and constraints. When the objective functions or constraints are complex, we may turn to heuristic algorithms or dynamic programming method, yet the computational burden is a potential problem for real-time control.

3.3. Artificial Intelligence-Based Models. Artificial intelligence (AI) is defined as the study of intelligent agents which perceives its environment and takes actions that maximize its chance of success at some goal. Traffic signal control based on $\mathrm{AI}$ is considered as a promising research area, because an AI system does not assume any prior knowledge of a model or the parameters of its dynamics and thus does not need to rely on the expensive and timeconsuming model calibration procedures like existing optimization model. Furthermore, the AI system is capable of "learning" from the environment and thus is expected to improve the operations over time and adapt to changes of the environment. This has been proved by some leading researches in recent years $[64,65]$. This type of models is very applicable to the traffic signal control under CV environment for the reason that the traffic information can be perceived in real time. Examples of AI approaches used in reviewed studies include multiagent system, fuzzy logic, neural network, and reinforcement learning.

Both vehicles and traffic signal controllers can be deemed as "agent," named as vehicle agent and control agent. In this perspective, the whole signal control system becomes a socalled multiagent system. The multiagent system used in a lot of studies has advantages including being model-free and coordinated, colearning, and being suitable for parallel processing. Khamis and Gomaa [66] developed an adaptive multiobjective reinforcement learning system for traffic signal control based on a cooperative multiagent framework. In addition, the authors show that using the Bayesian probability interpretation to estimate the parameters of the MDP probabilities can result in a good response to the traffic nonstationarity. Lee and Park [38] proposed a cooperative vehicle intersection control (CVIC) algorithm based on multiagent model that does not require a traffic signal. By eliminating the potential overlaps of vehicular trajectories coming from all conflicting approaches at the intersection, the CVIC algorithm seeks a safe maneuver for each vehicle approaching intersection and manipulates each of them. Also, an additional algorithm was designed to deal with the system failure cases resulting from inevitable trajectory overlaps at the intersection and infeasible solutions. A simulation-based case study implemented on a hypothetical 
four-way single-lane approach intersection under varying congestion conditions showed that the CVIC algorithm significantly improved intersection performance compared with conventional actuated intersection control: $99 \%$ and $33 \%$ of stop delay and total travel time reductions, respectively, were achieved. Kari et al. [23] developed an agentbased online adaptive signal control (ASC) strategy. The system demonstrated savings of $5-14 \%$ in reducing travel time and $0-5 \%$ in reducing system-wide fuel economy in a scenario with constant demand profiles compared with the QEM/HCM-based strategy. Other representative studies include $[67,68]$.

Reinforcement learning (RL) is a machine learning paradigm, by which a controller's policy can be optimized through trial-and-error interactions with an environment. The agent-environment interaction can be described as follows. At every time step $t$, the agent obtains the state of environment $s_{t}$. Given $s_{t}$, the agent will decide the next action $a_{t}$. Then the environment transits to a new state, $s_{t+1}$, for which a reward $r_{t+1}$ is given to the agent. The goal of reinforcement learning problem is to find the optimal policy which yields the highest total reward. A schematic illustration is shown in Figure 1. Reinforcement learning is particularly well suited to problems which include a longterm versus short-term reward trade-off. It has been applied successfully to various problems, including robot control, elevator scheduling, telecommunications, backgammon, checkers, and go (AlphaGo).

Liu et al. [69] presented distributed cooperative reinforcement learning-based traffic control that integrates V2X networks' dynamic clustering algorithm. A dynamic clustering algorithm is proposed based on the enhanced affinity propagation. By integrating the clustering algorithm, a cooperative reinforcement learning control scheme is proposed to balance the traffic load. To address the tough dimensionality curse of reinforcement learning, a distributed mechanism for intersection cooperation is introduced, and a fast gradient-descent function approximation method is proposed to improve the controls' real-time performance. The proposed algorithm gets the minimum intersection waiting time, serves the most road users, and produces the minimum queue length compared to the baselines. Cheng et al. [44] applied reinforcement learning to fine-tune the control parameters of the network and make it adaptive to various traffic conditions. The algorithm has good performance, especially in high dynamic traffic flows. Yang and Tan [70] provided some results on how the reinforcement learning method performs using $Q$-matrix and Q-network.

Wang et al. [71] formulated the joint traffic signal and connected vehicle control problem as a reinforcement learning (RL) problem, the action and state spaces of which are specifically designed to take into account the connected vehicles. An effective rewarding mechanism is designed, which takes into account the impact of the detouring on the network traffic efficiency. By utilizing tools from deep RL, an efficient algorithm is proposed to jointly control the traffic signals and the connected vehicles. Numerical results demonstrate validity and efficiency of the models.

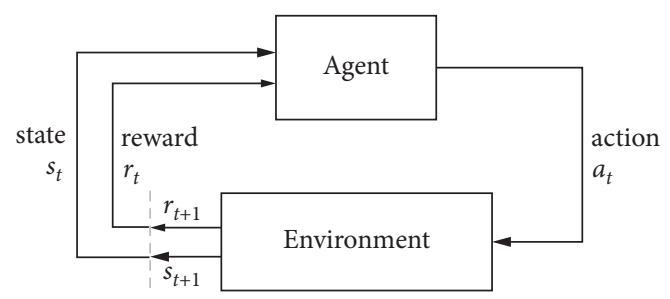

FIGURE 1: Schematic figure for reinforcement learning.

Another commonly used intelligent-control model is fuzzy logic-a mathematical system that analyzes analog input values in terms of logical variables that take on continuous values between 0 and 1 , in contrast to classical or digital logic, which operates on discrete values of either 1 or 0 (true or false, respectively). Collotta et al. [33] proposed a multicontroller system consisting of a Wireless Sensor Network (WSN), a Phase Sorting Module, and a fuzzy logic controller. The WSN is responsible for the collection of traffic data; the Phase Sorting Module determines the phase execution sequence; each fuzzy controller determines the green time duration for the relevant phase considering the number of enqueued cars in the lanes that are under its control. The fuzzy control strategy is also adopted in [44]. The authors determined the appropriate groups based on real-time traffic conditions using neurofuzzy network.

Before AI-based signal control system is widely implemented, it still faces significant hurdles, despite a large number of studies. A key problem is the lack of interpretability for AI algorithms, especially machine learning algorithms. In addition, some of AI algorithms require tremendous computing power.

3.4. Other Models. Ahmane et al. [45] proposed a model based on Timed Petri Nets with Multipliers (TPNM) which can make the control policy through the structural analysis. The control aims to smooth the traffic through the sequence of vehicles authorized to traverse the intersection. The proposed control policy is based on the modeling of an isolated 4-way intersection as a discrete event dynamic system. The dynamic behavior of the modeled traffic is discrete and represented by a Timed Petri Net with Multipliers (TPNM) in which multipliers are associated with the arcs of the Petri Net. Cheng et al. [72] used the gametheoretic paradigm of fictitious play to iteratively search for a coordinated signal timing plan to be employed, which improves a system-wide performance criterion for a traffic network. Elhenawy et al. [5] proposed a game-theory-based algorithm for controlling autonomous vehicle movements at uncontrolled intersections.

\section{Major Technical Issues}

4.1. Different Stages of $\mathrm{CV}$ Technologies. According to whether or not autonomous driving technology is equipped, connected vehicles can be divided into manned and automated types. The two types need to be controlled in different ways: manned connected vehicles might be still controlled 
using traffic signals just as conventional vehicles that cannot communicate with the central controller or other vehicles, while autonomous vehicles can be controlled by coordinated route planning without any traffic signal. In most of the reviewed studies such as $[22,36,57]$, the connected vehicles only refer to the manned connected vehicles. There are also studies that aim to solve the route planning problem in a fully autonomous vehicle environment $[38,44]$. The situation of two types of vehicles together with conventional vehicles has not been fully studied. One example is the study of [73], in which three categories of vehicles are considered: (1) conventional vehicles, (2) automated vehicles, and (3) manned connected vehicles. The study integrated three different stages of technology development and developed heuristics to switch the signal controls depending on the stage of technology. The simulation results show an evident decrease in the total number of stops and delay when using the connected-vehicle algorithm for the tested scenarios with information level as low as $50 \%$.

Rafter et al. [74] proposed a novel traffic signal control algorithm called Multimode Adaptive Traffic Signals (MATS) which can offer reductions in mean delay for networks with $0-100 \%$ connected-vehicle presence. The MATS algorithm combines position information from connected vehicles with data obtained from existing inductive loops and signal timing plans in the network to perform decentralized traffic signal control at urban intersections. The MATS algorithm is capable of adapting to scenarios with low numbers of connected vehicles, an area where existing traffic signal control strategies for connected environments are limited.

4.2. Centralized Control versus Decentralized Control. Majority of signal control systems use centralized formulation and architecture. In such systems, vehicles approaching the intersection communicate with a central controller at the intersection. The central controller optimizes various signal timing parameters of the system at the same time in one mathematical program. However, network signal timing optimization is known as an NP-complete problem and a central optimization technique will not be scalable and applicable to large transportation networks.

The other category of methods uses decentralized control (also called distributed control). Compared with centralized system, the paradigm decomposes the signal timing optimization problem to several interconnected subproblems in different control nodes. Each node only needs to execute a very simple computation, which reduces the complexity of each individual node. For example, Nafi et al. [75] presented a VANET-based road traffic signaling system developed using a distributed architecture by incorporating the distributed networking feature. Ishlam et al. [76] presented a Distributed-Coordinated methodology for signal timing optimization in connected urban street networks, with underlying assumption that all vehicles and intersections are connected, and intersections can share information with each other. The novelty of the work is the decentralized approach, where a mathematical program controls the timing of only a single intersection, which means the approach is in real time and scalable. The results show that the algorithm can increase intersection throughput between 1\% and $5 \%$ and reduce travel time between $17 \%$ and $48 \%$, compared to actuated coordinated signals. Decentralized architecture is also adopted in $[5,37,51]$. But the distributed system has also disadvantages: using distributed cooperative control completely could cause delays in realizing the traffic balance among districts. Therefore, a potential direction is introducing a hierarchical structure to incorporate the centralized control and the distributed cooperative control.

\subsection{Isolated Control versus Coordinated Control. There are} two distinct modes of traffic signal controller operation: isolated and coordinated. Many studies focus on isolated control because this mode is the foundation of intersection signal control and a significant part of coordinated mode. Liang et al. [77] developed a flexible, real-time traffic signal control algorithm to optimize both phase durations and phase sequences at four-approach intersections with conflicting left turns, based on information obtained from connected vehicles. The location of all connected vehicles is used to identify the presence of nonconnected vehicles that are stopped at the intersection and then identify naturally occurring platoons in the traffic stream. The signal control algorithm then selects the optimal sequence that these platoons should discharge through the intersection to minimize average delay of all identified vehicles. Several heuristic methods are proposed to determine optimal platoon departure sequences in this scenario. Other examples include $[22,37,47]$.

The goal of coordinated control is to achieve the global optimum in an arterial or a network, through considering the coordination of all the controllers. Wang et al. [78] developed a joint control model which optimizes the speeds of the connected vehicles and coordinating signals along an arterial simultaneously. This control model forms connected vehicles into platoons so that the vehicles can pass through intersections together with no stops or the least stop time. At the same time, it optimizes signal timing plans along an arterial to achieve lower signal delay and higher throughput. A real-world road network simulation shows that the joint control model can reduce the stop time and stops of coordinate phase by up to $53.69 \%$ and $41.15 \%$. The signalized intersection delay per vehicle is reduced by $13.19 \%$. Other representative studies include $[39,68]$.

\subsection{Analysis of Scenarios of CV Deployment with Different} Penetration Rates. The quality of traffic signal control under $\mathrm{CV}$ environment depends mainly on the number of vehicles equipped with communication devices with respect to the total number of vehicles, the so-called penetration rate. Therefore, various penetration rates should be modeled to obtain the impact factor exactly. He et al. [39] evaluated the performance of the proposed PAMSCOD system and concluded that the system can outperform state-of-practice signal control methods at about a $40 \%$ penetration rate. Feng et al. [57] presented a real-time adaptive signal phase 
allocation algorithm using connected-vehicle data. Due to the low penetration rate of the connected vehicles, an algorithm, EVLS, which estimates the states of unequipped vehicle based on connected-vehicle data is developed to construct a complete arrival table for the phase allocation algorithm. Evaluation shows that the proposed control algorithm outperforms actuated control by reducing total delay by as much as $16.33 \%$ in a high penetration rate case and similar delay in a low penetration rate case. Guler et al. [47] found that increase in the penetration rate from $0 \%$ up to $60 \%$ can significantly reduce the average delay for the proposed algorithm. The above-mentioned studies reveal that the existing control models can outperform the traditional control methods only in a relatively high penetration rate of connected vehicles, which may not be feasible in the near future. How to utilize real-world CV data under low penetration rate environment to improve traffic signal operation is a pressing issue. One of the related studies is that of Zheng and Liu [79]. The authors modeled vehicle arrivals at signalized intersections as a time-dependent Poisson process. An expectation maximization (EM) procedure is derived to solve the parameter estimation problem.

4.5. Priority Control. Priority control aims to improve service and reduce delay for certain traffic mode at intersections. The most common form is transit signal priority (TSP) control. Hu et al. [59] proposed a person-delay-based optimization method for an intelligent TSP logic TSPCV-C that enables bus/signal cooperation and coordination among consecutive signals under the CV environment. The method is evaluated through a computer simulation as well as in the field [60]. A similar form is freight signal priority (FSP). Rather than considering simply travel time and reliability, it is possible to consider vehicle weight, road grade, and truck engine type in order to minimize energy and emissions along a freight corridor [23].

The concept can be generalized for a system in which different traffic modes have different priority level. He et al. [39] presented a unified platoon-based formulation called PAMSCOD to concurrently optimize network traffic signal control for different travel modes given the assumption that advanced communication systems are available between vehicles and traffic controllers. Two modes of traffic composition (transit buses and passenger vehicles) are considered in a decision framework. Microscopic simulation shows that the proposed algorithm can successfully coordinate traffic signals considering the two traffic modes including buses and automobiles and significantly reduce vehicle delay for both modes. The algorithm was improved by the authors in another paper [58]. Liang et al. [80] proposed an algorithm that leverages information from connected vehicles (CVs) arriving at an intersection to identify naturally occurring platoons that consist of both CVs and non-CVs. Simulation tests reveal that the proposed platoon-based algorithm provides superior computational savings (over 95\%) compared with algorithms that focus on individual vehicles.
4.6. Performance Evaluation. There are four major elements in the performance evaluation for traffic control optimization: (1) measure of effectiveness (MOE), (2) evaluation platform, (3) evaluation scenarios, and (4) baselines. The widely used MOEs include average delay, stops, queue length, and energy consumption. Most studies use simulation software as evaluation platform such as VISSIM, SUMO, GLD, AIMSUN NG, and Commuter for microscopic traffic simulation and ns2, ns3, NCTUns, and OPNET for network communication simulation. Simulation experiments with different scenarios are carried out by covering varying volume-to-capacity ratios and market penetration rates in most studies. The baselines are usually classic algorithms such as Webster's method, TRANSYT, HCM method, and Synchro's optimization method.

It is noted that the car-following model in a connectedvehicle environment may be different from traditional models so appropriate adjustments of the default settings in traffic simulation software are necessary. However, no details about the adjustments are mentioned in the existing studies.

\section{Summary}

To demonstrate the development status of the research area more clearly, we summarize the basic information of the reviewed papers in Table 1.

\section{Perspectives of Future Development}

Connected vehicle is a promising technology which provides more detailed traffic information to optimize time planning of signalized intersections in real time. It has been proved that the introduction of $\mathrm{CV}$ technology into traffic signal control has potential in improving the road traffic flow efficiency, providing enhanced safety, saving energy consumption, and reducing pollutant emissions. The paper reviews the existing studies about time signal optimization under $\mathrm{CV}$ environment to illustrate the current state of the art in this research field. A variety of optimization models have been proposed and evaluated, achieving satisfying performance in either simulation or implementation. However, it is clear that the application of CV technology in the traffic signal optimization domain is still in its early stages. There remain many problems that are waiting to be solved. In this section, we raise unresolved problems and give our perspectives of the future research directions.

Under CV environment, the traffic flow characteristics can be quite different from those in current road traffic environment. Furthermore, things may become more complex due to the coexistence of conventional vehicles and manned connected vehicles together with connected autonomous vehicles. Therefore, the flow pattern under $\mathrm{CV}$ environment needs to be taken into account. In addition, signal control optimization can be incorporated with the help of driver assistant system in such environment to further reduce delays.

The current studies adopt either centralized or decentralized (or distributed) paradigm to optimize signal time 


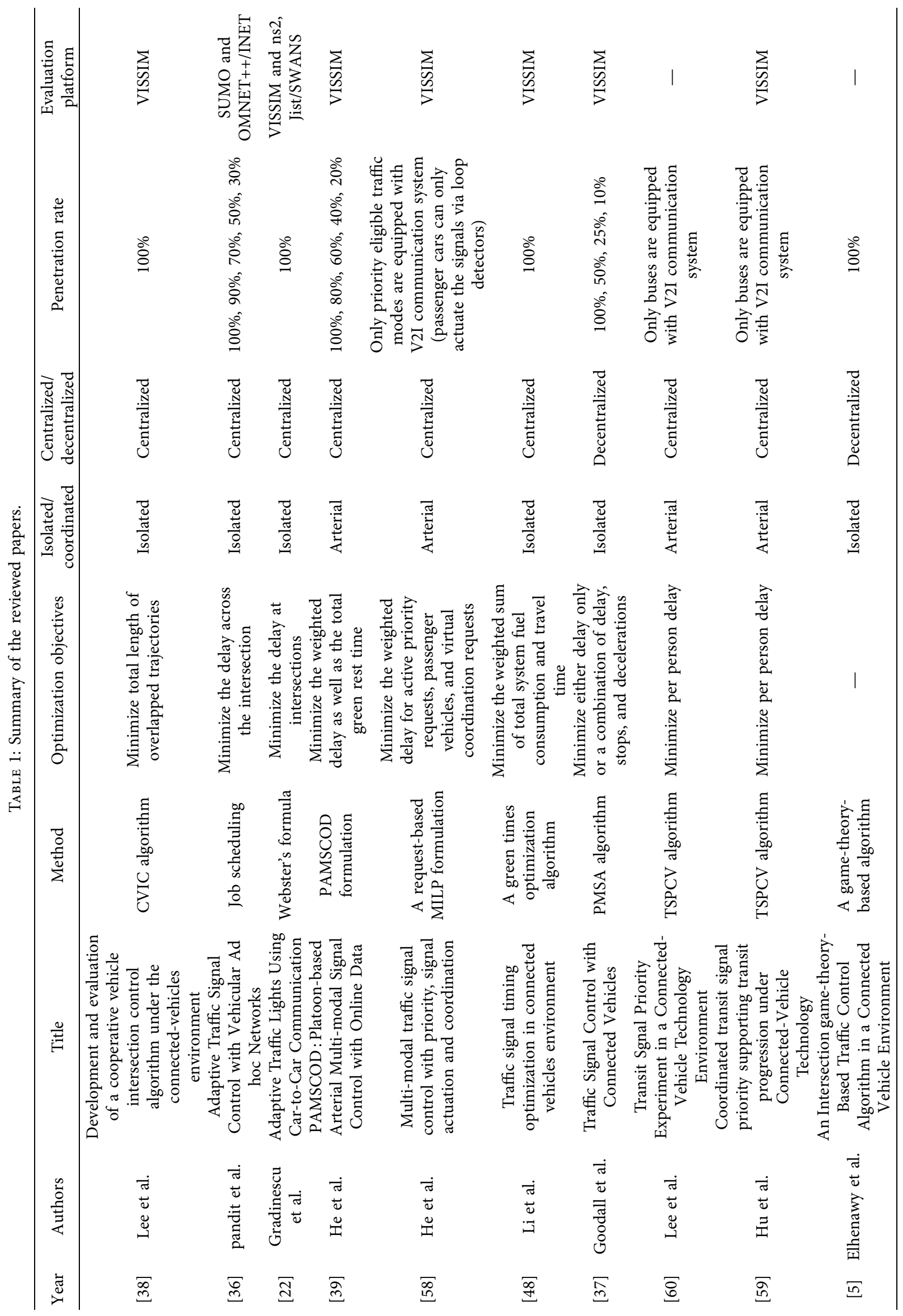




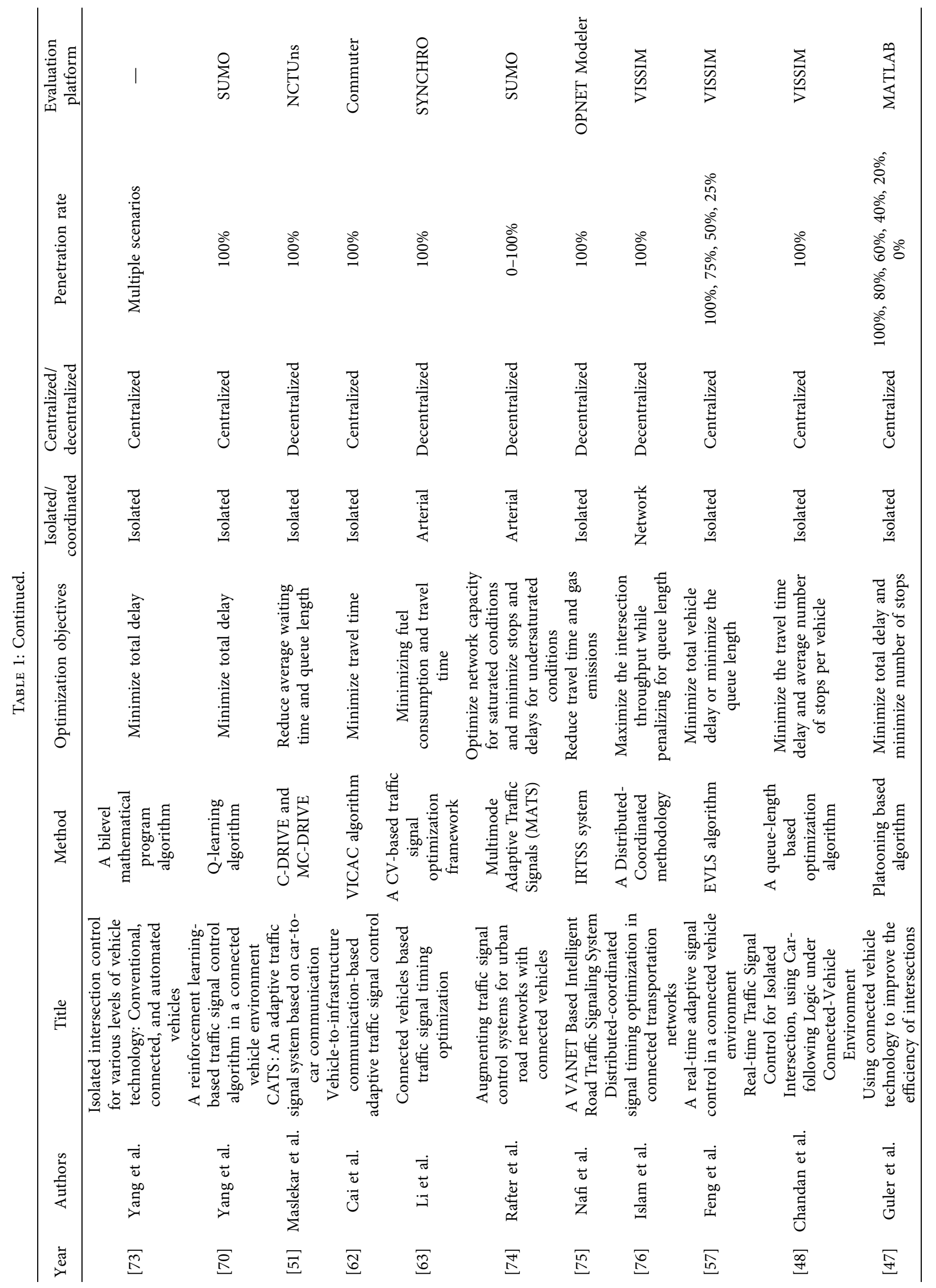




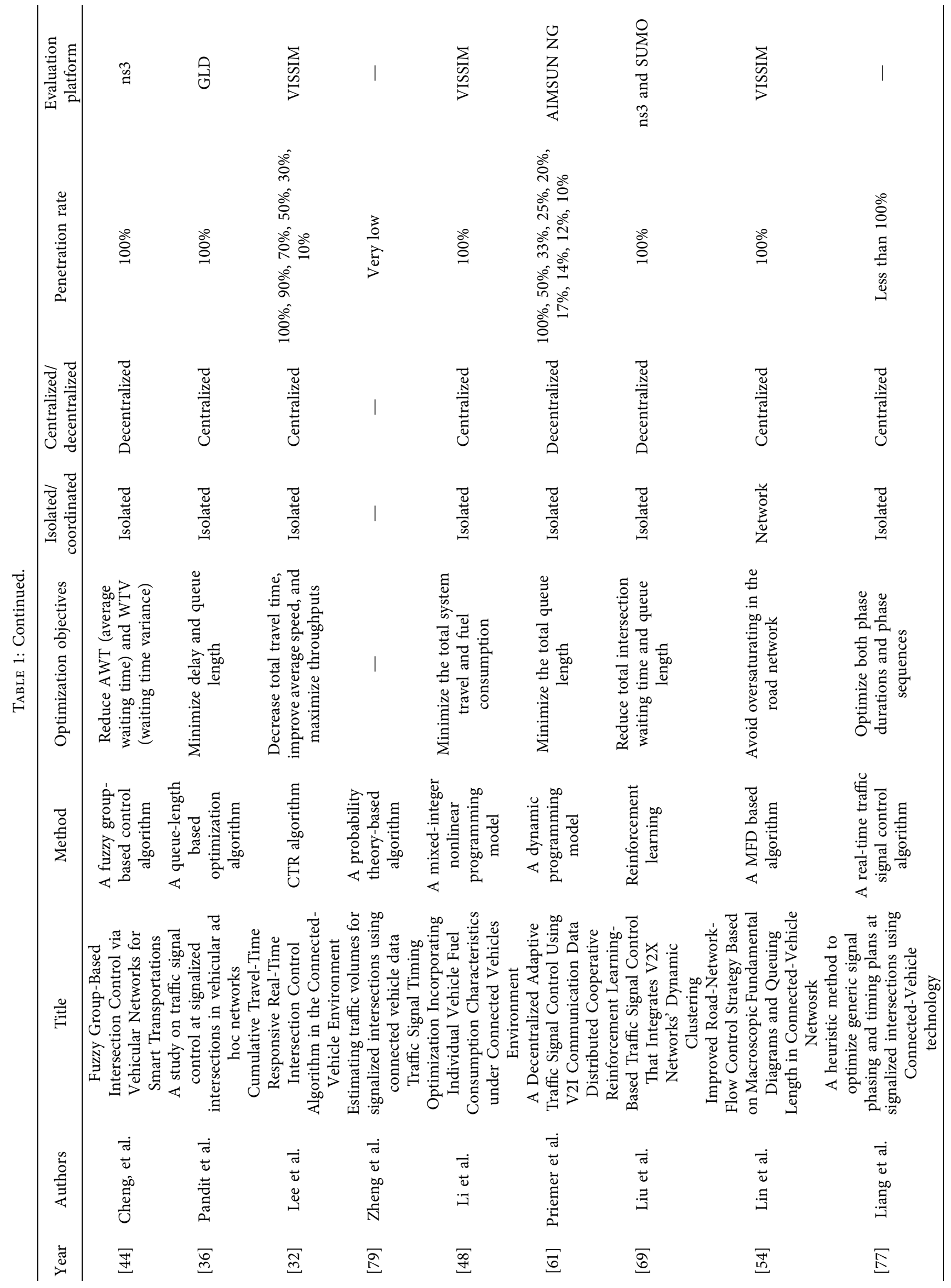




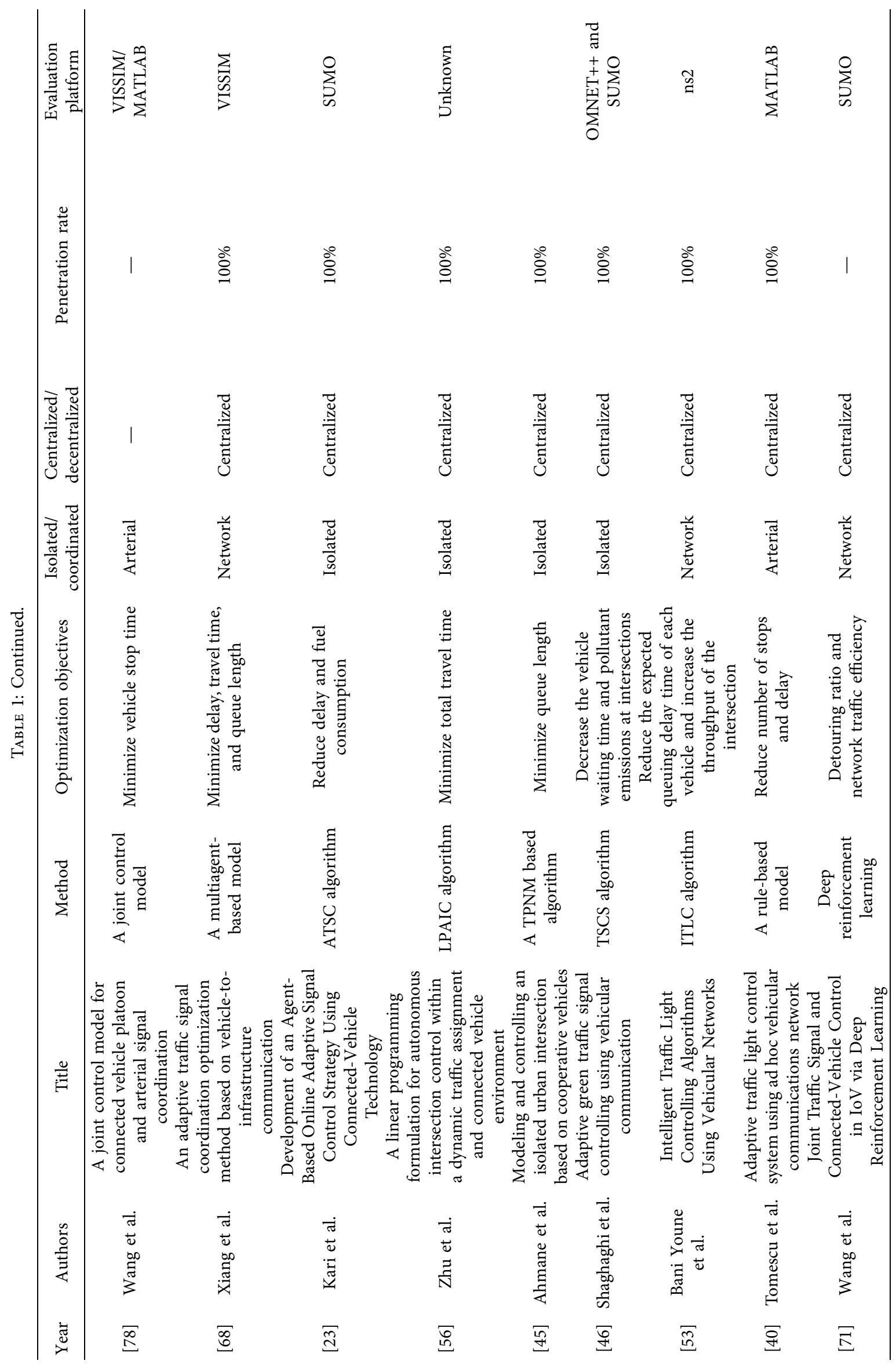




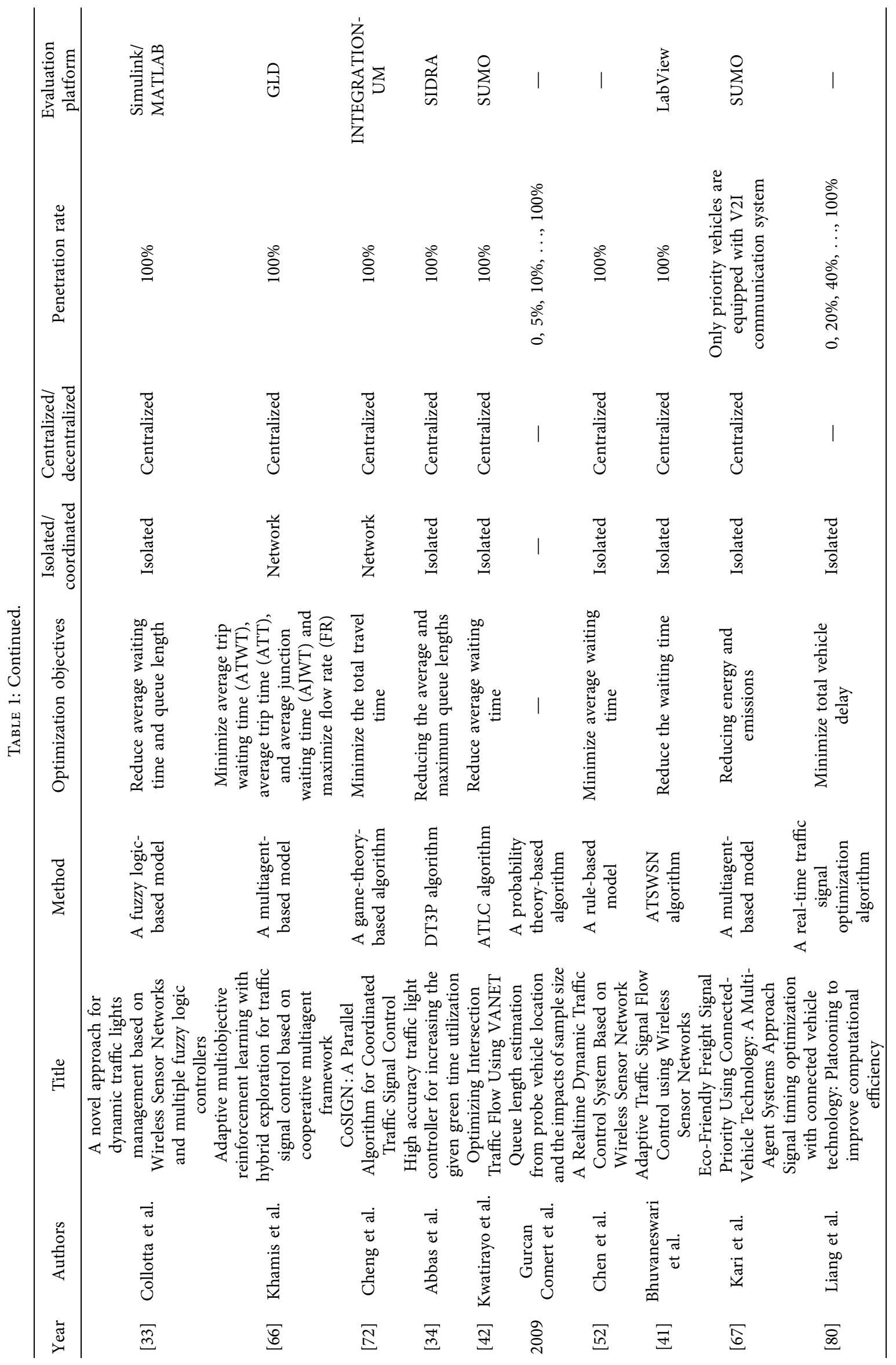


planning. Centralized control can achieve the coordination of different intersections easily, leading to a global optimal solution, yet the formulation might be computation-expensive in a large road network. In contrast, decentralized control is more scalable but inefficient in realizing coordination. The future traffic signal control system should incorporate the advantages of the two modes to achieve general applicability and higher efficiency.

Under the connected-vehicle environment, the greatest strength is the complete perception of the traffic states in the road network. The cooperation of different intersections, therefore, should be improved. But the study of networkwide signal optimization is insufficient. We take network control under CV environment as an important research direction.

Despite the rapid popularity of CV technology, the situation of imperfect penetration rate may last for a long time. Thus, the state estimation of unequipped vehicles (especially in a low penetration rate) will be a crucial problem at present. Although Zheng et al. [79] proposed a probability theory-based model to deal with the problem, the sphere of application of the method is still limited.

As shown in several studies, for example, [37], the algorithm can improve performance compared to a state-ofpractice coordinated actuated timing plan at low- and midlevel volumes, but performance worsens at oversaturated condition. The optimization strategies for oversaturated traffic are expected to be solved under CV environment.

In recent years, the field of artificial intelligence has achieved great breakthrough with the advance of deep learning and reinforcement learning. It is not surprising that the artificial intelligence algorithm has been introduced into traffic signal control and used for solving complex control optimization problems to which mathematical or conventional modeling is unlikely to be useful. We consider the artificial intelligence-based control as the future trend of traffic signal optimization.

\section{Data Availability}

All the reviewed papers in the manuscript are listed in Table 1.

\section{Conflicts of Interest}

The authors declare no conflicts of interest.

\section{Acknowledgments}

This study was jointly supported by Shanghai Science and Technology Committee, China (YDZX20193100004845, 21DZ205100, and 19DZ1208700).

\section{References}

[1] D. Schrank, B. Eisel, T. Lomax, and J. Bak, "Urban mobility report," Inrix, vol. 81, 2015.

[2] NHTSA, "Traffic safety facts 2015: a compilation of motor vehicle crash data from the fatality analysis reporting system and the general estimates system," U.S. Department of
Transportation - NHTSA - DOT HS 809 322, 2015, http:// www.trb.org/main/blurbs/172686.aspx.

[3] M. Desai and R. P. Harvey, "Inventory of U.S. Greenhouse gas emissions and sinks: 1990-2015. Fed," Register, vol. 82, Article ID 10767, 2017.

[4] A. Pizam, "Life and tourism in the year 2050," International Journal of Hospitality Management, vol. 18, pp. 331-343, 1999.

[5] M. Elhenawy, A. A. Elbery, A. A. Hassan, and H. A. Rakha, "An intersection game-theory-based traffic control algorithm in a connected vehicle environment," in Proceedings of the 2015 IEEE 18th International Conference on Intelligent Transportation Systems, pp. 343-347, Gran Canaria, Spain, September 2015.

[6] F. V. Webster and B. M. Cobbe, "Traffic signals," vol. 56, H. M. S. O., London, UK, 1966, Road Research Technique Paper.

[7] R. E. Allsop, "SIGSET: a computer program for calculating traffic signal settings," Traffic Engineering and Control, vol. 13, pp. 58-60, 1971.

[8] R. E. Allsop, "SIGCAP: a computer program for assessing the traffic capacity of signal-controlled road junctions," Traffic Engineering and Control, vol. 17, 1976.

[9] J. D. C. Little, M. D. Kelson, and N. H. Gartner, "MAXBAND: a program for setting signals on arteries and triangular networks," Transportation Research Record Journal of the Transportation Research Board, vol. 795, pp. 40-46, 1981.

[10] D. I. Robertson, "TRANSYT method for area traffic control," Traffic Engineering and Control, vol. 10, pp. 181-182, 1969.

[11] N. H. Gartner and R. M. Deshpande, "Dynamic programming approach for arterial signal optimization," Transportation Research Record: Journal of the Transportation Research Board, vol. 2356, no. 1, pp. 84-91, 2013.

[12] R. A. Vincent and J. R. Peirce, "MOVA" - experience and developments in signal control," in Proceedings of the PTRC TRAFFEX '93 Conference Proceedings," Seminar On Traffic Control: Control Systems, Information And Enforcement, National Exhibition Centre, Birmingham, UK, April 1993.

[13] P. Koonce, L. Rodegerdts, K. Lee et al., Traffic Signal Timing Manual, 2008.

[14] A. J. Miller, "A computer control system for traffic networks," in Proceedings of the International Symposium on the Theory of Traffic Flow and Transportation, London, UK, 1963.

[15] P. B. Hunt, D. I. Robertson, R. D. Bretherton, and R. I. Winton, SCOOT-A Traffic Responsive Method of Coordinating Signals, Transport and Road Research Laboratory, London, UK, 1981.

[16] A. G. Sims, "The Sydney coordinated adaptive traffic system," in Proceedings of the Engineering Foundation Conference on Research Directions in Computer Control of Urban Traffic Systems, Pacific Grove, CA, USA, February 1979.

[17] J. J. Henry, J. L. Farges, and J. Tuffal, "The PRODYN real time traffic algorithm," Control in Transportation Systems, pp. 305-310, 1984.

[18] N. H. Gartner, "OPAC: a demand-responsive strategy for traffic signal control," IFAC Proceedings Volumes, vol. 93, pp. 241-244, 1990.

[19] V. Mauro and D. Di Taranto, "UTOPIA: proceedings of the 6th IFAC/IFIP," in Proceedings of the IFORS Symposium on Control and Communication in Transportation, Paris, France, September 1990.

[20] P. Mirchandani and F.-Y. Wang, "RHODES to intelligent transportation systems," IEEE Intelligent Systems, vol. 20, pp. 10-15, 2005.

[21] M. M. Zanjireh and H. Larijani, "A survey on centralised and distributed clustering routing algorithms for WSNs," in 
Proceedings of the Vehicular Technology Conference, pp. 1-6, Boston, MA, USA, September 2015.

[22] V. Gradinescu, C. Gorgorin, R. Diaconescu, V. Cristea, and L. Iftode, "Adaptive traffic lights using car-to-car communication," in Proceedings of the 2007 IEEE 65th Vehicular Technology Conference - VTC2007-Spring, pp. 21-25, Dublin, Ireland, April 2007.

[23] D. Kari, G. Wu, and M. J. Barth, "Development of an agentbased online adaptive signal control strategy using connected vehicle technology," in Proceedings of the 17th International IEEE Conference on Intelligent Transportation Systems (ITSC), Shandong, China, October 2014.

[24] NHTSA, Preliminary Statement of Policy Concerning Automated Vehicles, National Highway Traffic Safety Administration, Washington, DC, USA, 2013.

[25] Y. Xie, H. Zhang, N. H. Gartner, and T. Arsava, "Collaborative merging strategy for freeway ramp operations in a connected and autonomous vehicles environment," Journal of Intelligent Transportation Systems, vol. 21, no. 2, pp. 136-147, 2017.

[26] Y. Wang, W. E, W. Tang, D. Tian, G. Lu, and G. Yu, "Automated on-ramp merging control algorithm based on Internet-connected vehicles," IET Intelligent Transport Systems, vol. 7, no. 4, pp. 371-379, 2013.

[27] R. A. Uzcátegui, A. J. De Sucre, and G. Acosta-Marum, "Wave: a tutorial," IEEE Communications Magazine, vol. 47, 2009.

[28] D. Llusia, R. Márquez, J. F. Beltrán, C. Moreira, and J. P. D. Amaral, "IEEE 802.11p: towards an international standard for wireless access in vehicular environments," in Proceedings of the Vehicular Technology Conference, pp. 2036-2040, Calgary, Canada, September 2008.

[29] D. Committee, "Dedicated short range communications (DSRC) message set dictionary," Technical Report J2735_ 200911, Society of Automotive Engineers, Warrendale, PA, USA, 2009.

[30] R. Braun, F. Busch, C. Kemper et al., "TRAVOLUTION-Netzweite Optimierung der Lichtsignalsteuerung und LSAFahrzeug-Kommunikation," Stra\{ß\}enverkehrstechnik, vol. 53, pp. 365-374, 2009.

[31] BMW, BMW GREEN Wave Project, [WWW Document]. URL http://www.themotorreport.com.au/46137/bmwpushing-for-smarter-adaptive-traffic-lights, 2009.

[32] J. Lee, B. Park, and I. Yun, "Cumulative travel-time responsive (CTR) real-time intersection control algorithm under the connected vehicle environment," Journal of Transportation Engineering, vol. 139, Article ID 130605045950002, 2013.

[33] M. Collotta, L. Lo Bello, and G. Pau, "A novel approach for dynamic traffic lights management based on Wireless Sensor Networks and multiple fuzzy logic controllers," Expert Systems with Applications, vol. 42, no. 13, pp. 5403-5415, 2015.

[34] M. K. Abbas, M. N. Karsiti, M. Napiah, B. B. Samir, M. AlJemeli, and H. Dag, "High accuracy traffic light controller for increasing the given green time utilization," Computers \& Electrical Engineering, vol. 41, pp. 40-51, 2015.

[35] S. M. A. B. A. Islam, A. Hajbabaie, H. M. A. Aziz, and H. M. A. Aziz, "A real-time network-level traffic signal control methodology with partial connected vehicle information," Transportation Research Part C: Emerging Technologies, vol. 121, Article ID 102830, 2020.

[36] K. Pandit, C. Chuah, H. M. Michael, and D. Ghosal, "Adaptive traffic signal control with vehicular ad hoc networks," IEEE Transactions on Vehicular Technology, vol. 62, pp. 1459-1471, 2013.
[37] N. J. Goodall, B. L. Smith, and B. Park, "Traffic signal control with connected vehicles," Transportation Research Record: Journal of the Transportation Research Board, vol. 2381, no. 1, pp. 65-72, 2013.

[38] J. Lee and B. Park, "Development and evaluation of a cooperative vehicle intersection control algorithm under the connected vehicles environment," IEEE Transactions on Intelligent Transportation Systems, vol. 13, no. 1, pp. 81-90, 2012.

[39] Q. He, K. L. Head, and J. Ding, "PAMSCOD: platoon-based arterial multi-modal signal control with online data," Transportation Research Part C: Emerging Technologies, vol. 20, no. 1, pp. 164-184, 2012.

[40] O. Tomescu, I. M. Moise, and A. E. Stanciu, "Adaptive traffic light control system using ad hoc vehicular communications network," UPB Scientific Bulletin, Series D, vol. 74, no. 2, 2012.

[41] P. T. V. Bhuvaneswari, G. V. A. Raj, R. Balaji, and S. Kanagasabai, "Adaptive traffic signal flow control using wireless sensor networks," in Proceedings of the 2012 Fourth International Conference on Computational Intelligence and Communication Networks, pp. 85-89, Uttar Pradesh, India, November 2012.

[42] S. Kwatirayo, J. Almhana, and Z. Liu, "Optimizing intersection traffic flow using VANET," in Proceedings of the 2013 IEEE International Conference on Sensing, Communications and Networking (SECON), pp. 260-262, New Orleans, LA, USA, June 2013.

[43] Z. Yao, Y. Jiang, B. Zhao, X. Luo, and B. Peng, "A dynamic optimization method for adaptive signal control in a connected vehicle environment," Journal of Intelligent Transportation Systems, vol. 24, no. 2, pp. 184-200, 2020.

[44] J. Cheng, W. Wu, J. Cao, and K. Li, "Fuzzy group-based intersection control via vehicular networks for smart transportations," IEEE Transactions on Industrial Informatics, vol. 13, pp. 751-758, 2017.

[45] M. Ahmane, A. Abbas-Turki, F. Perronnet et al., "Modeling and controlling an isolated urban intersection based on cooperative vehicles," Transportation Research Part C: Emerging Technologies, vol. 28, pp. 44-62, 2013.

[46] E. Shaghaghi, M. R. Jabbarpour, R. Md Noor, H. Yeo, and J. J. Jung, "Adaptive green traffic signal controlling using vehicular communication," Frontiers of Information Technology and Electronic Engineering, vol. 18, no. 3, pp. 373-393, 2017.

[47] S. I. Guler, M. Menendez, and L. Meier, "Using connected vehicle technology to improve the efficiency of intersections," Transportation Research Part C: Emerging Technologies, vol. 46, pp. 121-131, 2014.

[48] K. Chandan, W. Li, X. J. Ban, and J. Wang, "Traffic signal timing optimization incorporating individual vehicle fuel consumption characteristics under connected vehicles environment," in Proceedings of the 2016 International Conference on Connected Vehicles and Expo (ICCVE), pp. 13-18, Seattle, WA, USA, September 2016.

[49] A. Hajbabaie and R. F. Benekohal, "Traffic signal timing optimization: choosing the objective function," Transportation Research Record Journal of the Transportation Research Board, vol. 2355, no. 19, 10 pages, 2013.

[50] F. V. Webster, "Traffic signal settings. road research," Department of Scientific and Industrial Research, London, UK, Technical Paper NO. 39, 1958.

[51] N. Maslekar, J. Mouzna, M. Boussedjra, and H. Labiod, "CATS: an adaptive traffic signal system based on car-to-car communication," Journal of Network and Computer Applications, vol. 36, no. 5, pp. 1308-1315, 2013. 
[52] W. Chen, L. Lifeng Chen, Z. Zhanglong Chen, and S. Shiliang $\mathrm{Tu}$, "A realtime dynamic traffic control system based on wireless sensor network," in Proceedings of the 2005 International Conference on Parallel Processing Workshops (ICPPW'05), vol. 1, pp. 258-264, Oslo, Norway, June 2005.

[53] M. Bani Younes and A. Boukerche, "Intelligent traffic light controlling algorithms using vehicular networks," IEEE Transactions on Vehicular Technology, vol. 65, no. 8, pp. 5887-5899, 2016.

[54] X. Lin, J. Xu, P. Lin, C. Cao, and J. Liu, "Improved roadnetwork-flow control strategy based on macroscopic fundamental diagrams and queuing length in connected-vehicle network," Mathematical Problems in Engineering, vol. 2017, Article ID 8784067, 7 pages, 2017.

[55] H. P. Williams, Model Building in Mathematical Programming, John Wiley and Sons, New York, NY, USA, 2013.

[56] F. Zhu and S. V. Ukkusuri, "A linear programming formulation for autonomous intersection control within a dynamic traffic assignment and connected vehicle environment," Transportation Research Part C: Emerging Technologies, vol. 55, pp. 363-378, 2015.

[57] Y. Feng, K. L. Head, S. Khoshmagham, and M. Zamanipour, "A real-time adaptive signal control in a connected vehicle environment," Transportation Research Part C: Emerging Technologies, vol. 55, pp. 460-473, 2015.

[58] Q. He, K. L. Head, and J. Ding, "Multi-modal traffic signal control with priority, signal actuation and coordination," Transportation Research Part C: Emerging Technologies, vol. 46, pp. 65-82, 2014.

[59] J. Hu, B. B. Park, and Y.-J. Lee, “Coordinated transit signal priority supporting transit progression under connected vehicle technology," Transportation Research Part C: Emerging Technologies, vol. 55, pp. 393-408, 2015.

[60] Y.-J. Lee, S. Dadvar, J. Hu, and B. B. Park, "Transit signal priority experiment in a connected vehicle technology environment," Journal of Transportation Engineering, Part A: Systems, vol. 143, no. 8, Article ID 05017005, 2017.

[61] C. Priemer and B. Friedrich, "A decentralized adaptive traffic signal control using V2I communication data," in Proceedings of the 2009 12th International IEEE Conference on Intelligent Transportation Systems, pp. 765-770, St. Louis, MO, USA, October 2009.

[62] C. Cai, Y. Wang, and G. Geers, "Vehicle-to-infrastructure communication-based adaptive traffic signal control," IET Intelligent Transport Systems, vol. 7, no. 3, pp. 351-360, 2013.

[63] W. Li and X. Ban, "Connected vehicles based traffic signal timing optimization," IEEE Transactions on Intelligent Transportation Systems, vol. 20, no. 12, pp. 4354-4366, 2018.

[64] E. V.D. Pol, "Deep reinforcement learning for coordination in traffic light control," Master Thesis, Faculteit der Natuurwetenschappen, Wiskunde en Informatica, Amsterdam, Netherlands, 2016.

[65] L. Li, Y. Lv, and F.-Y. Wang, "Traffic signal timing via deep reinforcement learning," IEEE/CAA Journal of Automatica Sinica, vol. 3, no. 3, pp. 247-254, 2016.

[66] M. A. Khamis and W. Gomaa, "Adaptive multi-objective reinforcement learning with hybrid exploration for traffic signal control based on cooperative multi-agent framework," Engineering Applications of Artificial Intelligence, vol. 29, pp. 134-151, 2014.

[67] D. Kari, G. Wu, and M. J. Barth, "Eco - friendly freight signal priority using connected vehicle technology: a multi - agent systems approach," in Proceedings of the 2014 IEEE Intelligent
Vehicles Symposium Proceedings, pp. 1187-1192, Dearborn, MI, USA, June 2014.

[68] J. Xiang and Z. Chen, "An adaptive traffic signal coordination optimization method based on vehicle-to-infrastructure communication," Cluster Computing, vol. 19, no. 3, pp. 1503-1514, 2016.

[69] W. Liu, G. Qin, Y. He, and F. Jiang, "Distributed cooperative reinforcement learning-based traffic signal control that integrates V2X networks' dynamic clustering," IEEE Transactions on Vehicular Technology, vol. 66, no. 10, pp. 8667-8681, 2017.

[70] K. Yang and I. Tan, "A reinforcement learning based traffic signal control algorithm in a connected vehicle environment," in Proceedings of the 17th Swiss Transport Research Conference, Ascona, Switzerland, May 2017.

[71] Z. Wang, H. Zhu, Y. Zhou, and X. Luo, "Joint traffic signal and connected vehicle control in IoV via deep reinforcement learning," in Proceedings of the 2021 IEEE Wireless Communications and Networking Conference (WCNC), pp. 1-6, IEEE, Najing, China, April 2021.

[72] S.-F. Cheng, M. A. Epelman, and R. L. Smith, "CoSIGN: a parallel algorithm for coordinated traffic signal control," IEEE Transactions on Intelligent Transportation Systems, vol. 7, no. 4, pp. 551-564, 2006.

[73] K. Yang, S. I. Guler, and M. Menendez, "Isolated intersection control for various levels of vehicle technology: conventional, connected, and automated vehicles," Transportation Research Part C: Emerging Technologies, vol. 72, pp. 109-129, 2016.

[74] C. B. Rafter, B. Anvari, S. Box, and T. Cherrett, "Augmenting traffic signal control systems for urban road networks with connected vehicles," IEEE Transactions on Intelligent Transportation Systems, vol. 21, no. 4, pp. 1728-1740, 2020.

[75] N. S. Nafi and J. Y. Khan, "A VANET based intelligent road traffic signalling system," in Proceedings of the Australasian Telecommunication Networks and Applications Conference (ATNAC) 2012, Brisbane, Australia, September 2012.

[76] S. M. A. B. A. Islam and A. Hajbabaie, "Distributed coordinated signal timing optimization in connected transportation networks," Transportation Research Part C: Emerging Technologies, vol. 80, pp. 272-285, 2017.

[77] X. Liang, S. I. Guler, and V. V. Gayah, "A heuristic method to optimize generic signal phasing and timing plans at signalized intersections using connected vehicle technology," Transportation Research Part C: Emerging Technologies, vol. 111, pp. 156-170, 2020.

[78] P. Wang, Y. Jiang, L. Xiao, Y. Zhao, and Y. Li, “A joint control model for connected vehicle platoon and arterial signal coordination," Journal of Intelligent Transportation Systems, vol. 24, no. 1, pp. 81-92, 2020.

[79] J. Zheng and H. X. Liu, "Estimating traffic volumes for signalized intersections using connected vehicle data," Transportation Research Part C: Emerging Technologies, vol. 79, pp. 347-362, 2017.

[80] X. Liang, S. I. Guler, and V. V. Gayah, "Signal timing optimization with connected vehicle technology: platooning to improve computational efficiency," Transportation Research Record: Journal of the Transportation Research Board, vol. 2672, no. 18, pp. 81-92, 2018. 